\title{
Biomedical Waste Disposal Practices in a Tertiary Level Hospital - An Effort to Bridge the Gap Between Policy and Reality
}

\section{Prerna Gupta* and SK Verma}

Department of Medicine, Pacific Medical College and Hospitals, Udaipur, Rajasthan, India

*Corresponding Author: Prerna Gupta, Department of Medicine, Pacific Medical College and Hospitals, Udaipur, Rajasthan, India.
Received: December 20, 2021

Published: December 29, 2021

(C) All rights are reserved by Prerna Gupta and SK Verma.

\begin{abstract}
Objectives: The objectives of the study were to evaluate the infrastructural setup for Bio-medical waste (BMW) handling, segregation and mutilation at the point generation of the waste and to identify the shortcomings in the execution of the same in a tertiary level hospital in Udaipur, Rajasthan (India) in the month of September and October, 2021 with the help of a standard checklist with 22 questions and scoring out of 22.

Background: Establishing policies and ensuring its effective execution in a hospital are two completely different goals to achieve, when it comes to biomedical waste (BMW) handling, segregation and mutilation. The careless handling of BMW has a potential to become a major health hazard affecting community especially health workers. This study is a critical reminder that the protocols for BMW handling should be followed efficiently and in a strictly monitored environment to achieve the ideal management of BMW. There is always an enormous scope to know about the challenges faced in day to day execution of biomedical waste disposal.

Methods: The cross-sectional study was conducted quantitatively among 17 specialties and 5 clinical labs in a tertiary level hospital in Udaipur, Rajasthan (India) in the month of September, 2021 to October, 2021.The approach followed in this study was that, a standard checklist of 22 questions was prepared keeping in mind the regulations related to BMW. Each response appropriate according to Bio-Medical Waste (Management and Handling) Rules, 2016 was denoted in scoring as 1, otherwise as 0. So, the scoring was done out of 22. Data was further interpreted by graphs and percentages, and the outcomes were subsequently analyzed.

Results: Results included major problems such as- failure of mutilation of intact needles, failure to follow color coding, uncovered bins, poor hygiene, absence of particular color coded bins, unattended blood bags and failure to segregate in few of the places. Among the poorest scoring specialty includes Neurology outpatient department (OPD), COVID OPD (swab sample collection room), Dialysis treatment room, Neuroscience semi deluxe ward and sample collection room.

Conclusion: This is an example of a study conducted to evaluate the condition of hospital waste management in a tertiary level hospital. There is a further scope to study about the knowledge and practices of BMW Management among health workers to identify where these shortcomings in the execution stem from. Further, studies like this should be conducted in large numbers among health care workers and tertiary level hospitals to know the real status of BMW disposal system in order to improve the quality of waste management.
\end{abstract}

Keywords: Biomedical Waste; Waste Management; Segregation

\section{Introduction}

"Bio-medical waste" means any waste, which is generated during the diagnosis, treatment or immunisation of human beings or animals or research activities pertaining thereto or in the production or testing of biological or in health camps [1]. It comprises of $10-25 \%$ of healthcare waste which is hazardous, create a variety of health risk [2]. 
Types of waste according to WHO [3]

1. Infectious waste: Waste contaminated with blood and other bodily fluids (e.g. from discarded diagnostic samples), cultures and stocks of infectious agents from laboratory work (e.g. waste from autopsies and infected animals from laboratories), or waste from patients with infections (e.g. swabs, bandages and disposable medical devices)

2. Pathological waste: Human tissues, organs or fluids, body parts and contaminated animal carcasses;

3. Sharps waste: Syringes, needles, disposable scalpels and blades, etc.

4. Chemical waste: For example solvents and reagents used for laboratory preparations, disinfectants, agents used for sterilisation and heavy metals contained in medical devices (e.g. mercury in broken thermometers) and batteries

5. Pharmacy waste: Expired, unused and contaminated drugs and vaccines

6. Cytotoxic waste: Waste containing substances with genotoxic properties (i.e. highly hazardous substances that are, mutagenic, teratogenic or carcinogenic), such as cytotoxic drugs used in cancer treatment and their metabolites

7. Radioactive waste: Such as products contaminated by radionuclides including radioactive diagnostic material or radio therapeutic materials

8. Non-hazardous or general waste: Waste that does not pose any particular biological, chemical, radioactive or physical hazard.

Biomedical waste must be properly managed and disposed of to protect the environment, general public and workers, especially healthcare and sanitation workers who are at risk of exposure to biomedical waste as an occupational hazard. Steps in the management of biomedical waste include generation, accumulation, handling, storage, treatment, transport and disposal [4]. Examples of disease spread by hospital waste include respiratory infections, gastro enteric infections, genital infections, ocular infection, skin infections, acquired immunodeficiency syndrome (AIDS), meningitis, hemorrhagic fevers, anthrax, septicemia, viral hepatitis A, bacteremia, candidaemia, viral hepatitis B and C [5].

\section{Objectives}

The objectives of the study were to evaluate the infrastructural setup for BMW handling, segregation and mutilation at the point generation of the waste and to identify the shortcomings in the exe- cution of the same in a tertiary level hospital in Udaipur, Rajasthan (India) in the month of September and October, 2021 with the help of a standard checklist with 22 questions and scoring out of 22 .

\section{MATERIAL and METHODS:}

The cross-sectional study was conducted quantitatively among 17 specialties and 5 clinical labs in a tertiary level hospitalin Udaipur, Rajasthan (India) in the month of September, 2021 to October, 2021.

The primary data has been collected with the help of a standardized checklist mentioned below [6,7] followed meticulously at all the places of study. The checklist was prepared in a way that it could be answered in a binary form, either yes or no. While following the format strictly, the places of study were analyzed. Pilot study was not conducted.

The approach followed in this study was that, each response appropriate according to Bio-Medical Waste (Management and Handling) Rules, 2016 should be denoted in scoring as 1, otherwise as Zero. The scoring was done out of 22 that is the total number of questions in the checklist given below:

- Is red color bin available?

- Is yellow color bin available?

- Is blue color bin available?

- Is black color bin available?

- Is white translucent color puncture-proof container available?

- Is red color bag placed in the red color bin?

- Is yellow color bag placed in the yellow color bin?

- Is blue color sticker/mark placed in the blue color bin?

- Is black color bag placed in the black color bin?

- Does the white translucent color container contain 5\% sodium hypochlorite solution?

- Is the biohazard symbol printed over waste bags/bins?

- $\quad$ Are the color bins covered?

- Any blood splashes?

- $\quad$ Does the red bin with red bag contain only plastics?

- $\quad$ Does the yellow bin with yellow bag contain only soiled infectious waste?

- Does the blue bin with blue sticker/mark contain only glass(broken or unbroken), metallic and body implants? 
- $\quad$ Does the white translucent color container containing 5\% sodium hypochlorite solution contain only infected sharps?

- Does the black bin with black bag contain general waste?

- Is used hypodermic needle kept unattended on table/trolley/counter?

- Is used hypodermic needle destroyed?

- Is used hypodermic needle disposed in white punctureproof containers?

- Is used hypodermic needle re-capped?

The data was subsequently interpreted in the form of a graph and percentages of score, scored by each specialty and lab. Further, analysis of the outcomes was done in form of observations and discussions.

\section{Observations and Results}

Most common and the most concerning observation was the failure of mutilation of used needles in Emergency Wards, Blood Bank (Figure 1) and Dialysis treatment rooms (Figure 2). Although there was presence of needle destroyer and cutter, but still the intact needles/recapped needles were being collected in a container for disposal. The above resulted in frequent needle stick injury.

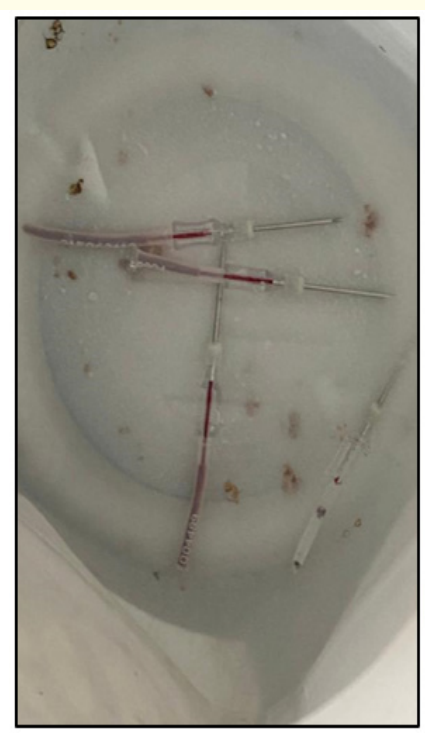

Figure 1: Needles not destroyed [Blood bank (donation room)].

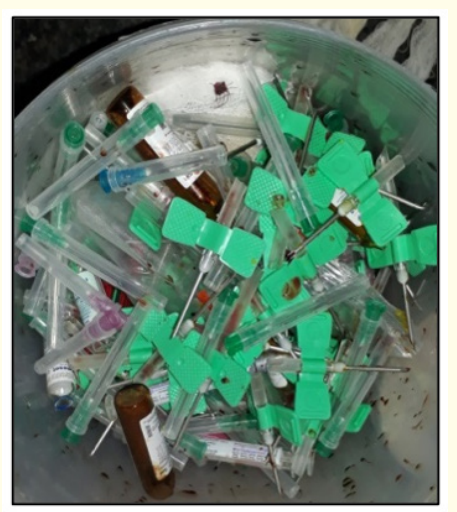

Figure 2: Intact and recapped needles and broken ampules with blood splashes [Dialysis- Treatment Room].

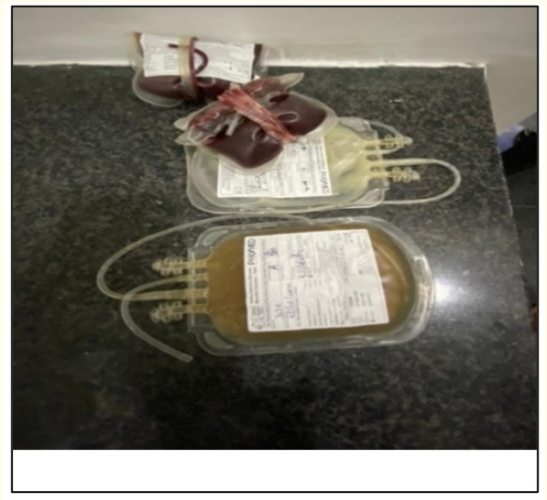

Figure 3: Unattended blood bags (Blood Bank- sterilization room).

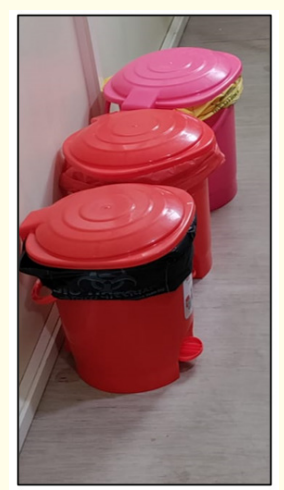

Figure 4: Incorrect Color Coding [MICU]. 
Other observations included

- $\quad$ Presence of non-foot operated lids of the bins.

- Unattended blood bags lying on the counter in sterilization room at blood bank (Figure 3)

- Uncovered bins in Radio-diagnostic department, COVID OPD (swab sample collection area) and Ortho OPD (Plaster room).

- Incorrect color coding of bins and bags in Emergency wards, COVID OPD (swab sample collection area), Chemotherapy ward, MICU (Figure 4) and Sample collection room.

- Non- availability of some color coded bins in Neurology outpatient department (OPD), Sample collection room and COVID OPD (swab sample collection area).

- Most of the bins or areas did not have visible biohazard sign.

- $\quad$ Blood splashes on wall and trays in blood bank.

The data in the graph given below suggests a strong relationship between low scores and significant deficiencies found in the execution of handling, segregation and mutilation of BMW (Figure 5).
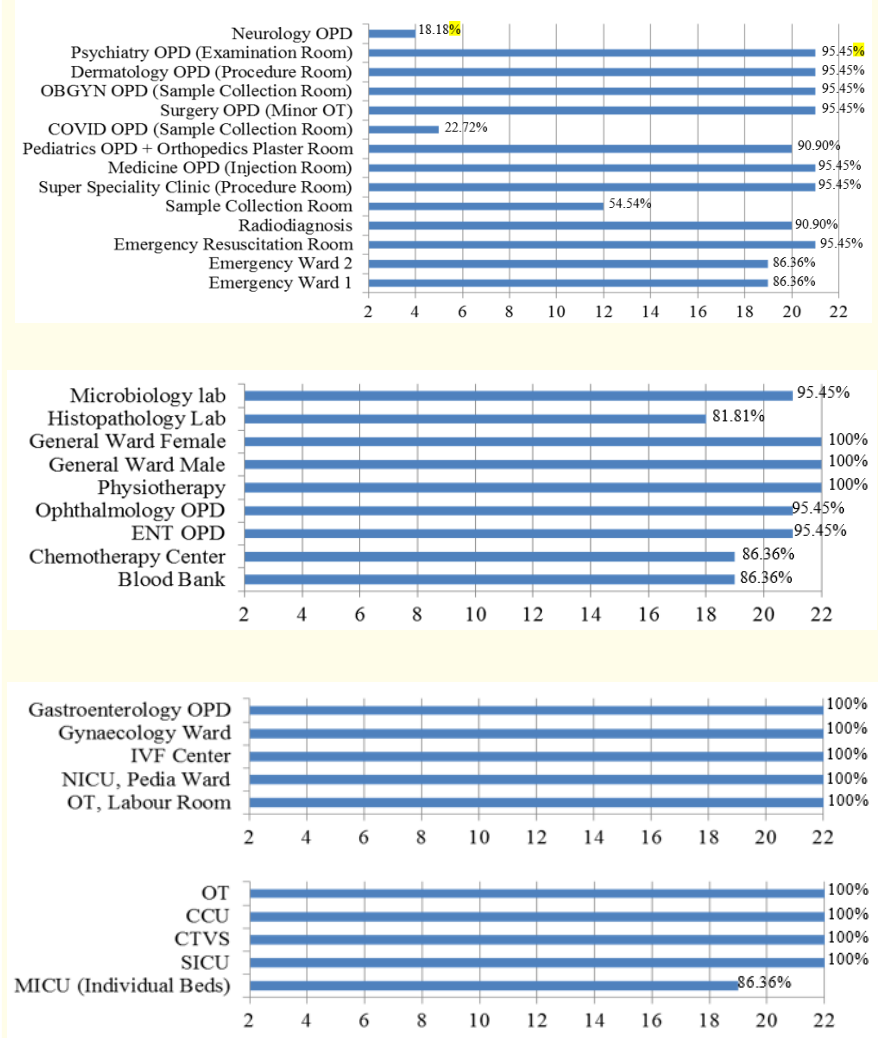

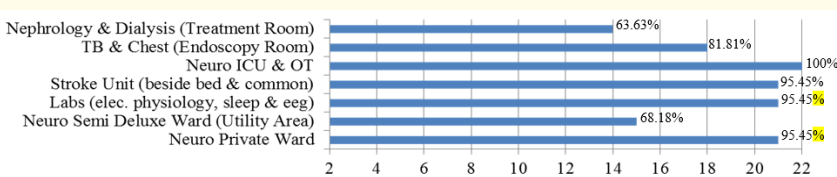

Figure 5: Graph showing specialties and labs score out of 22 (in percentage).

Looking at the graph above, it can be observed how Neurology outpatient department scored $18.18 \%$ due to lack of different color coded bins, justified by the staff as "we don't do any procedures here in opd". But when investigated there were pieces of blood soiled gauges found in the black bin which itself defeated the whole purpose of segregation.

Sample collection room just scored $54.54 \%$ because the room only had a red bin. It had several sky blue colored small bins with black bags that violated color coding and the rest of the bins were missing.

Further, COVID OPD (swab sample collection area) scored $22.72 \%$ because it had an uncovered yellow bin and none of the other bins were found. The same scenario was observed at blood sample collection room where no color coding was followed and the bins being used were also not covered.

One of the most astonishing finding was that, one of the most well equipped areas namely Neuroscience semi deluxe ward that scored $68.18 \%$ and dialysis treatment room that scored $63.63 \%$ had a very poor condition of waste management. Broken ampules, used blades, blood soiled gauges, intact/recapped needles, blood splashes on container and incorrect color coded bins lying around were one of the few examples of failed execution of proper segregation and storage of waste.

Some of the other places like endoscopy room in TB and Chest department had bins containing wrong category of waste. Most of the other places either had incorrect color coding or wrong category of waste disposal.

\section{Discussion}

A total of 17 specialties and 5 labs in the tertiary level hospital were included in this study. The top sources contributing in biomedical waste generation in this tertiary level hospital at Udaipur 
are Wards, Labs, Operation Theatres, Blood Bank, Chemotherapy Center, Dialysis Center and Intensive Care Units.

Proper management of Biomedical waste (BMW) generated in a healthcare facility is one of the most important functions of a healthcare worker (HCW) as its improper management not only poses risk to human beings and environment, but may also invite legal action against HCW as well as hospital administration [8].

According to the findings of the study, following are some recommendations addressed to the hospital administration to bridge the gaps and deficiencies:

- Waste bins should have foot-operated lids.

- The bins should contain the matching colours of bag and a clearly visible biohazard sign.

- Importance needs to be given for 'mutilation of waste' especially in wards like needles should always be cut or burnt before putting in 5\% sodium hypochlorite solution.

- Every out patient department should have all colour coded bins whether or not procedures are done there and at all times of the day.

- $\quad$ Proper use of BMW colour coding should be enforced to promote ideal segregation practices.

- The bins should be covered at all times of the day.

- Periodic sensitisation of health-care workers and housekeeping staff should be done consistently to emphasize on the importance of usage of personal protective equipment to avoid needle stick injury.

- $\quad$ Periodic inspection of the BMW generating areas by authorities and creating a sense of accountability among personnel involved in BMW Management.

In a 600-bedded super-specialty corporate hospital of a South Indian city, there were only white receptacles for all types of BMW for aesthetic reasons and since the color of all receptacles or bins was same, following the segregation practices was difficult [8]. On the contrary, in the current study, 5 types of colored bins mainly yellow, red, white, blue and black were present.

Various reports indicate that the implementation of the BMW Rules is not satisfactory even in the large towns and cities in India [9]. Looking at the results of the survey from Irbid city of Jordan, it can be observed that the healthcare facilities in the city have less appropriate practices when it comes to the handling, storage, and disposal of wastes generated in comparison to the developed world. There were no defined methods for handling and disposal of these wastes. No specific regulations or guidelines for segregation or classification of the wastes were established, that lead to mixing of BMW with general waste [10]. On the other hand in India, we already have a well-established Bio-Medical Waste (Management and Handling) Rules, 2016 in effect. Also, in the current study segregation was found to be followed far better than the healthcare facilities in Irbid city of Jordan.

The arrangements for bulk clinical waste handling were audited in 16 UK hospitals, one year after an earlier audit that revealed many deficiencies in performance. Storage of clinical waste carts in areas accessible to members of the public and failure to lock individual waste carts was common. Waste segregation was poor. Many clinical waste carts and the areas dedicated to their storage were in a poor state of repair. Many instances of clinical waste storage apparently in breach of UK health and safety legislation, of fire regulations, and of the hazardous waste regulations were observed [11]. In the current study, there were uncovered bins found. Also, many of the bins did not have a biohazard sign visible on it due to poor condition on the bin.

The current place of study has a better performance than the places mentioned in the referenced studies. Still, there is a great scope to fill the gaps between policies and reality with the help of recommendations above.

\section{Conclusion}

The present study was done to evaluate the infrastructural setup for BMW handling, segregation and mutilation at the point generation of the waste and to identify the shortcomings in the execution of the same in a tertiary level hospital in Udaipur, Rajasthan (India) based on a standardised checklist. The above study made us realise how ground-level execution of waste management is a challenging task. It doesn't only involve formation of rules and regulations but enormously depends on how it is followed in real time. There is a further scope to study about the knowledge and practices of BMW Management among health workers to identify where these shortcomings in the execution stem from. This is an example of a study conducted to evaluate the condition of hospital waste management in a tertiary level hospital. Further, studies like this should be con- 
ducted in large numbers among health care workers and tertiary level hospitals to know the real status of BMW disposal system in order to improve the quality of waste management.

\section{Acknowledgements}

We are sincerely thankful to Dr. (Air Commodore) S.C. Kabra, Dr. S.C. Vashishtha, Dr. S.K. Nagori and Mrs. Vishakha Parmar for guiding me throughout this project with their valuable inputs and to all the departments of the tertiary level hospital for their cooperation.

\section{Bibliography}

1. "Government of India ministry of environment, forest and climate change. Notification". New Delhi: (Bishwanath Sinha) Joint secretary to the Government of India (2016): 10-20.

2. Park K. "Park'S Textbook Of Preventive And Social Medicine". $25^{\text {th }}$ ed. Bhanot Publishers (2021): 849.

3. "Health-care waste". Who.int. (2018).

4. U.S. Congress, Office of Technology Assessment, Finding the Rx for Managing Medical Wastes, OTA-O-459 (Washington, DC: U.S. Government Printing Office, September) (1990).

5. Chandrappa R and Das D. "Biomedical Waste". Solid Waste Management. $1^{\text {st }}$ ed. Springer (2012): 147-175.

6. Ramalingam A and Saikumar C. "A study on evaluation of biomedical waste management in a tertiary care hospital in South India". Tropical Journal of Pathology and Microbiology 4.7 (2018): 520-521.

7. Gaur K. "Community Medicine Practical Guide". $2^{\text {nd }}$ ed. CBS publishers and distributors Pvt LTD; (2017): 163-174.

8. Kumar R., et al. "A descriptive study on evaluation of biomedical waste management in a tertiary care public hospital of North India". Journal of Environmental Health Science and Engineering 12.1 (2014).

9. Hanumantha Rao P. "Hospital waste management system - a case study of a south Indian city". Waste Management and Research 27.4 (2009): 313-321.

10. Bdour A., et al. "Assessment of medical wastes management practice: A case study of the northern part of Jordan". Waste Management 27.6 (2007): 746-759.

11. Blenkharn J. "Standards of clinical waste management in hospitals-A second look". Public Health 121.7 (2007): 540-545.

\section{Assets from publication with us}

- Prompt Acknowledgement after receiving the article

- Thorough Double blinded peer review

- Rapid Publication

- Issue of Publication Certificate

- High visibility of your Published work

Website: www.actascientific.com/

Submit Article: www.actascientific.com/submission.php

Email us: editor@actascientific.com

Contact us: +919182824667

Citation: Gupta P and Verma SK. "Biomedical Waste Disposal Practices in a Tertiary Level Hospital - An Effort to Bridge the Gap Between Policy and Reality". Acta Scientific Medical Sciences 6.1 (2022): 314-319. 